\title{
Accidentes de tránsito con heridos en Colombia según fuentes de información: caracterización general y tipologías de accidentes*
}

\section{Road traffic accidents with injured in Colombia according to information sources: General characterization and accident typologies}

\author{
José Ignacio Ruiz , Aura Nidia Herrera² \\ Universidad Nacional de Colombia
}

Forma de citar: Ruíz, J., \& Herrera, A. (2016). Accidentes de tránsito con heridos en Colombia según fuentes de información: caracterización general y tipologías de accidentes. Revista CES Psicología, 9(1), 32-46.

\begin{abstract}
Resumen
El objetivo de esta investigación es conocer las dinámicas sociales de la accidentalidad vial -formas de ocurrencia, tipos de consecuencias, tipos de víctimas- y su relación con variables sociodemográficas. Se analizan datos procedentes de dos fuentes: una encuesta de victimización vial (accidentalidad subjetiva) a una muestra no aleatoria de estudiantes de ciclos superiores de 20 ciudades capitales ( $\mathrm{n}=2292)$ de Colombia y estadísticas oficiales de heridos en tránsito (accidentalidad objetiva) en Bogotá en el año 2009 $(\mathrm{n}=2300)$. Mediante un análisis de clasificación jerárquica y combinando características del vehículo, del daño causado y del conductor -edad, sexo- y otros actores de la vía, los resultados mostraron cuatro tipologías de accidentes: 1) conductor particular, atropello, víctima peatón de 61 años o más, 2) víctima peatón de 0 a 10 años por atropello y lesión por trauma facial, 3) víctima pasajero o mujer, por caída desde el automóvil o bus de transporte público, y lesiones en el cuello, 4) víctima conductor de moto o cicla, entre 21 y 30 años, varón, por colisión, y daños en las extremidades. Las implicaciones de los resultados se discuten con relación a políticas de prevención de la accidentalidad vial.
\end{abstract}

Palabras clave: Accidentes de Tráfico, Accidentalidad Subjetiva, Accidentalidad Objetiva, Tipo de Daño, Tipo de Vehículo.

DOI: http://dx.doi.org/10.21615/cesp.9.1.3

* Esta investigación se enmarcó en el Proyecto Democracia, Seguridad Humana y Tejido Social financiado por la Facultad de Ciencias Humanas de la Universidad Nacional de Colombia, en la Convocatoria Orlando Fals Borda del 2010.

${ }^{1}$ Ph.D. en Psicología, Máster en Criminología, Profesor Asociado Departamento de Psicología, Universidad Nacional de Colombia. iiruizp@unal.edu.co

${ }^{2}$ Ph.D. en Diseños, evaluación y tecnología informática en ciencias del comportamiento. Laboratorio de Psicometría. Departamento de Psicología. Universidad Nacional de Colombia, Ciudad Universitaria, Bogotá. anherrerar@unal.edu.co 
José Ignacio Ruiz \& Aura Nidia Herrera

ACCIDENTES DE TRÁNSITO CON HERIDOS EN COLOMBIA SEGÚN FUENTES DE INFORMACIÓN: CARACTERIZACIÓN GENERAL Y TIPOLOGÍAS DE ACCIDENTES

\begin{abstract}
The aim of this paper is to describe the social dynamics of road traffic accidents - occurrence forms, consequences of accidents, victim typologies - and their relations with socio demographic variables. Data of two types were analyzed: the driving victimization survey (subjective traffic accidents) by a nonrandomized sample of high school students ( $\mathrm{n}=2292$ ) from 20 main Colombian cities and official statistics of traffic injured (objective traffic accidents) in Bogotá during $2009(n=2300)$. Through a cluster analysis and combining vehicle characteristics, caused damage and driver age- sex- and other actors of the road, the results showed four types of accidents: 1) personal vehicle driver, 61 year-old or older pedestrian fatality. 2) Pedestrian victim from 0 to 10 years old, injury by facial trauma. 3) Passenger or woman victim from public transportation or vehicle falling, neck injuries. 4) 21-30 year-old driver man of moto or bicycle, crash victim, leg and hand injured.
\end{abstract}

Key words: Subjective Driving Accidents, Driving Behavior, Objective Driving, Accidents, Transportation Accidents, Type of Injury, Type of Vehicle.

\section{Introdución}

El problema de la accidentalidad del tráfico vial no es nuevo. El criminólogo alemán Günther Kaiser dedicó en el año 1978 a este tema un denso volumen de la serie Estudios en Psicología Criminal que publicaba por aquellos años la editorial Espasa-Calpe a partir de la obra de autores tan relevantes en el campo de la criminología y la victimología como Von Henting o Middhendorf. En aquella ocasión, Kaiser se refería a que los avances de la tecnología y la ciencia habían traído consigo como aspectos negativos una serie de peligros, prácticamente desconocidos hasta entonces para la humanidad. Señalaba este autor que "uno de esos puntos peligrosos, aún no dominado, aunque no se desconoce su trascendencia, es el ocasionado por la circulación en las vías públicas" (Kaiser, 1978, p.25). También advertía que esta problemática no era percibida como tal por muchos ciudadanos, quizá por que muchos no se reconocen en el ámbito de la conducción como potenciales delincuentes y, por ello, se muestran más comprensivos hacia este tipo de infracciones.
Veinticinco años después de tal advertencia, en el año 2003, la Organización Mundial de la Salud (OMS) situaba los peligros asociados al tráfico rodado como una de las tres epidemias desatendidas y ocultas de nuestros tiempos, junto con el tabaquismo y las enfermedades cardiovasculares (OMS, 2003). Los datos al respecto, recogidos por la misma OMS y otras fuentes, son contundentes. En un informe sobre los traumatismos ligados al tránsito vial, la OMS (2009) informa que en el 2004, hubo más de 1,2 millones de víctimas mortales por accidentes de tránsito, afectando principalmente a las personas de 5 a 44 años de edad. Además, se estima que en el mundo se producen anualmente entre 20 y 50 millones de traumatismos no mortales por tránsito, y que en el 2030 la mortalidad por el tránsito será la quinta causa de muerte a nivel mundial, mientras que ahora ya es la novena, y morirán unos 2,4 millones de personas por esta causa, si no se llevan a cabo las políticas adecuadas. Este crecimiento de la accidentalidad y de sus múltiples consecuencias afectará más a los países de ingresos medios y bajos, mientras que en los de altos ingresos las tasas de accidentalidad vial comienzan a decrecer. 
El mismo informe indica que hay cinco factores de riesgo fuertemente asociados con la accidentalidad: 1) la conducción bajo los efectos del alcohol, 2) el exceso de velocidad, y la ausencia de sistemas de protección como 3) cascos, 4) cinturones de seguridad y 5) sistemas de protección para los niños.

En el caso de Colombia, la OMS, basándose en datos del Instituto de Medicina Legal y Ciencias Forenses, informa de 5409 fallecidos por accidentalidad vial en el 2001 (80\% hombres) y más de 38.000 víctimas no fatales en el año 2007; registra, sin embargo, un descenso en el número de muertes, de 20 casos por 100.000 habitantes en 1997 a alrededor de 15 casos por 20.000 habitantes en el 2007. Según Cesvi Colombia (2003) la accidentalidad vial era en el 2002 la segunda causa de mortalidad violenta en el país. En ese año, de 6063 muertos, el $39 \%$ eran peatones, el $19 \%$ pasajeros y el $15 \%$ motociclistas. El $75 \%$ de las víctimas fueron hombres, y estas diferencias entre sexos se mantienen hasta el momento.

Tanto las referencias a los principales factores de riesgo en la conducción, mencionados anteriormente, como los perfiles de victimas y de infractores del tránsito vial, muestran que el factor humano es el principal responsable de la accidentalidad vial (Montoro, Alonso, Esteban, \& Toledo, 2001). Factor humano que comprende, en los conductores, variables asociadas a la accidentalidad como el sueño, el cansancio, el estrés o el consumo de alcohol y otras sustancias psicoactivas (Montoro et al., 2001), la ira, la hostilidad, la percepción de la conducción como una tarea difícil (Ruiz E López, 2010; Ruiz, 2013) o las actitudes negativas hacia la conducción (Kaiser, 1978; Rodríguez, 2009; Sharkin, 2004; Ruiz, Beltrán, Gómez, Lamus, E Leal, 2014), el sentido de obligación de respetar las normas y ciertos sesgos en la percepción y acatamiento de éstas (Yagil, 2000) o las estrategias de búsqueda de espacios por donde cruza una vía, en el caso de peatones (Schwebel, Stavrinos, $\mathcal{E}$ Kongable, 2009). Tales estudios son abordados con frecuencia con muestras, representativas o no, y apelando a la medición de variables psicológicas, incluyendo la evocación del recuerdo de accidentes sufridos, por lo que podemos llamar a este enfoque de trabajo como dirigido a evaluar la accidentalidad subjetiva, frente a los datos procedentes de fuentes oficiales -ej.: policías de tránsito, institutos de medicina legal-, basados en el registro y conteo de casos, que se pueden denominar como de accidentalidad objetiva y que permiten realizar estudios de tipo censal, a partir de todos los casos registrados. Hay que tener en cuenta que es posible que para un mismo contexto haya discrepancia en datos y resultados de unas $\mathrm{u}$ otras fuentes de información accidentalidad objetiva y subjetiva-. Por ejemplo, las muestras quizá no son aleatorias y representativas de la población de donde fueron tomadas, al basarse en la colaboración voluntaria de quienes responden las encuestas, mientras que las cifras de los organismos oficiales de tránsito quizá no recogen aquellos accidentes, en general leves, en los cuales las partes involucradas llegan a un acuerdo sin que la autoridad registre el evento.

Estos registros oficiales de accidentalidad permiten analizar las dinámicas y diferencias en la accidentalidad vial entre hombres y mujeres, o entre grupos etáreos. Así, se ha concluido que la tasa de accidentalidad vial es mayor en hombres que en mujeres en el Reino Unido (Social Issues Research Centre, 2004), en España (OMS, 2002) y en Francia (Martin, Lafont, Chiron, Gadeqbeku, E Laumon, 2004), con unas diferencias de 3 a 1 en el caso de fallecimientos viales (Francia) o lesionados (España). Sin embargo, otros estudios ponen en entredicho la atribución de esa menor accidentalidad vial a las mujeres, al incluir entre la información analizada otras variables. Por 
ejemplo, Sivak y Schoettle (2011) identifican seis escenarios de accidentes viales choques entre vehículos- a partir de información sobre la dirección de aproximación entre los vehículos, su velocidad, el ángulo de colisión y el género de los conductores. Los autores hallaron que los choques entre mujeres estaban sobre representados en cinco de los seis escenarios, y los choques entre hombres estaban subrepresentados en cuatro escenarios, es decir ciertos accidentes son más probables entre mujeres y otros que se den entre hombres. En peatones, con relación a cruzar la vía aprovechando las brechas o espacios (gaps), se ha encontrado que tal conducta es menos probable en las mujeres (Koh E Wong, 2014) o que las mujeres emplean más tiempo para hacerlo (revisión de Kadali E Vedagiri, 2013), aunque el género no sea, en últimas, una variable significativa en los modelos predictivos de este tipo de conductas (Kadali \& Vedagiri, 2013; Koh E Wong, 2014).

Por otro lado, se ha estudiado las relaciones entre tipos de usuarios de las vías involucrados en accidentes, y el tipo de lesión. Por ejemplo, Mayou y Bryant (2003) compararon las consecuencias psicológicas, sociales y legales, y las condiciones físicas de peatones, motociclistas y automovilistas después de tres meses, uno año $\mathrm{y}$ tres años de haber sufrido accidentes viales, encontrando que, a corto plazo, los daños fueron mayores en los peatones y motociclistas, pero las diferencias entre los tres grupos de conductores se difuminaban en el seguimiento.

Este conjunto de estudios muestra que para entender el fenómeno del tráfico vial, tan necesario para la vida actual como impactante en el número de consecuencias fatales y lesivas que genera, se debe considerar multitud de factores, algunos de los cuales se han expuesto en los párrafos precedentes. Un primer paso, sin embargo, es conocer el nivel de accidentalidad real en nuestro contexto medido por el número de víctimas directas e indirectas que ocasiona la accidentalidad vial. Por ejemplo, es de suponer que así como ocurre en otros delitos, existe una cifra oscura de la accidentalidad vial, especialmente cuando ésta no deriva en consecuencias fatales heridos graves o fallecimientos-, por lo que el nivel de victimización puede ser superior a lo que reflejan las estadísticas oficiales. No obstante, dicha suposición dejará de serlo cuando se puedan comparar cifras y tasas obtenidas de ambas fuentes: estadísticas de denuncias y encuestas de victimización. Tal es el primer objetivo de este trabajo. El segundo consiste en analizar las relaciones entre características de los vehículos implicados en los accidentes viales, con las características de las víctimas y del tipo de daño sufrido por ellas a consecuencia de los eventos viales. Esta aproximación que permite identificar asociaciones entre perfiles de víctimas, victimarios y tipos de hechos, se ha empleado para estudiar otras conductas antijurídicas (ver Miethe E McCorkle, 2001; Morales, 2003).

\section{Metodología}

Este trabajo analiza la información sobre víctimas de accidentes de tránsito, provenientes de dos fuentes, por un lado, los datos recopilados mediante una investigación con estudiantes universitarios y del Sistema Nacional de Aprendizaje (SENA) de todo el país, realizada en el 2010, y por otro lado, los registros del Instituto de Medicina Legal y Ciencias Forenses de Colombia (en adelante, abreviado, INML) sobre heridos en accidentes de tránsito en el 2009 para la ciudad de Bogotá ( $n=2300)$. La elección de la base de datos de Medicina Legal obedeció a la disponibilidad y a la proximidad con la fecha en la que se realizó la encuesta. El diseño de esta investigación es exploratorio y comparativo, dentro de una perspectiva transversal de la recolección de datos. 
Base de datos sobre accidentalidad vial objetiva

Por razones de comparabilidad de los datos del INML se escogió para los análisis el grupo etáreo equivalente al de la muestra de participantes en la investigación. Los datos procedentes del INML recopilan las estadísticas de accidentalidad vial -heridos y muertos- en Bogotá en el año 2009. Para este trabajo se emplearon las estadísticas sobre heridos y se analizó la información sobre las siguientes variables: sexo (masculino, femenino), nivel educativo (ninguno, preescolar, primaria incompleta y completa, secundaria incompleta y completa, estudios superiores, técnicos), estado civil (soltero/a, unión libre, casado/a, divorcio o separación, viudo/a, religoso/a), zona del hecho (rural, urbana), escenario (calles, vía, vehículo particular, vehiculo servicio público, sin respuesta), actividad de la víctima durante el accidente (ir a actividades de ocio, descanso, traslado desde o al lugar de trabajo, quehacer habitual no remunerado, otras actividades), circunstancias o causas del accidente (embriaguez, exceso de velocidad, violación normas de peatones, violación otras normas de tránsito, otras causas, no consta de información), mecanismo de lesión (por determinar, abrasivo, contundente, cortante, corto-contundente), diagnóstico de lesión (politrauma, sin lesiones, trauma área pélvica, craneana, en abdomen, en cuello, en miembros, en torax, lesión facial).

Otras variables incluidas en los registros del INML fueron: tipo de vehículo (ambulancia, vehiculo, bicicleta, bus, buseta, camión tipo furgón, camioneta, campero, microbús, moto o motocarro, trasmilenio), tipo de accidente (atropello, caída del ocupante, choque con objeto fijo, colisión con otro vehículo, hundimiento, volcamiento), vehículo u objeto con el que se colisionó (automóvil, bicicleta, bus, buseta, camión furgón, camioneta, moto o motocarro, objeto fijo, otros), La variable víctima, por su lado, incluye las categorías: conductor, pasajero y peatón; y la variable edad se categorizó en ocho categorías: de 0 a 10 años, de 11 a 15, de 16 a 20, de 21 a 30, de 31 a 40 , de 41 a 50 y de 61 años en adelante.

\section{Participantes}

Se preguntó de forma anónima y colectiva a grupos de estudiantes si en los últimos seis meses les habían ocurrido, entre otros, los siguientes tres sucesos: 1) lesiones por accidente vial, 2) algún familiar o amigo había sido víctima de un accidente vial, y 3) algún familiar o amigo murió por accidente de tránsito. Los datos que se presentan sobre este estudio fueron recogidos entre septiembre y noviembre del 2010, conformando una muestra de 2292 personas entrevistadas en 20 ciudades de igual número de departamentos del país. La muestra fue intencional, basada en aplicación colectiva en salones de clase a estudiantes universitarios y del SENA que aceptaron participar en la encuesta, sin que recibieran algún tipo de contraprestación por ello. De todas las instituciones a las que se les propuso participar en la investigación contribuyendo a obtener una muestra, se trabajó con aquellas que aceptaron dicha colaboración. Las encuestas se aplicaron en instituciones colaboradoras de las capitales de departamento, que aceptaron colaborar en la investigación. Por ello, las instituciones participantes, especialmente las universidades, no son representativas de la población estudiantil de cada ciudad ni del departamento. Por otro lado, se hizo un esfuerzo en que en cada ciudad parte de la muestra fueran estudiantes del SENA, debido a que es una de las pocas instituciones educativas que tiene presencia en todos los departamentos del país, y ello ayudó a controlar la variable de tipo de institución 
educativa. Las preguntas hacían parte de una escala de victimización, que junto a escalas de percepción de tejido social y de democracia conformaban una batería de preguntas. Una panorámica de la investigación y análisis descriptivos de las escalas se presenta en Ruiz, López, Norza y Rodríguez (2012). En una investigación con otra escala de la batería se halló una correlación significativa de indicadores objetivos de criminalidad de la Policía Nacional a nivel departamental, con percepciones agregadas de las muestras por departamento en una escala de resiliencia comunitaria que hacía parte de la escala ( $\underline{\text { Ruiz, 2015) }}$, lo cual sirve de respaldo al uso de muestras de estudiantes para estudiar fenómenos colectivos de nivel nacional, aspecto también tratado por Páez y Vergara (2000). El 62,9\% de los sujetos fueron hombres, con una edad media de 22,12 años (DT: 4,63), y la mayoría solteros $(83,3 \%)$. En cuanto al nivel socioeconómico, el 89\% pertenecían a los estratos 1 a 3 (clase social muy baja a medio baja), y el 55\% eran estudiantes universitarios mientras que el resto tenían formación técnica o tecnológica.

Plan de análisis

En primer lugar, mediante el programa SPSS v. 15.0, se realizó un analisis descriptivo de las respuestas a la encuesta con estudiantes y se calculó y correlacionaron los tres indicadores de victimización vial: 1) lesiones personales por accidente de tránsito, 2) lesiones de familiar o amigo y 3) muerte de familiar o amigo, ocurridos dentro de los seis meses anteriores a la encuesta.

En segundo lugar, se analizó la información sobre variables sociodemográficas de quienes reportaron haber sido lesionados en accidentes viales y se compararon los datos de las dos fuentes -INML y encuesta a estudiantes. Para dicha comparación se seleccionaron submuestras de estudiantes que resultaran equiparables por edad y nivel educativo, con los datos del INML.

De otra parte, se analizaron las características de la accidentalidad vial con heridos para la base de datos del INML mediante estadísticos descriptivos de variables del accidente y de la víctima. En el primer caso se analizó la zona en que ocurrió el accidente y su posible causa, el tipo de vehículo y el tipo de accidente; las variables de la víctima fueron su rol (peaton, pasajero, conductor) en el accidente, la actividad que desarrollaba cuando éste ocurrió y el tipo de daño o dignóstico recibido como consecuencia del mismo. Además mediante el estadísitico chi2 para comparación de proporciones, se compararon los porcentajes de sujetos en cada categoría de las variables para grupos de sujetos según género y nivel educativo y edad.

Finalmente, se llevó a cabo con los datos del INML, un análisis de conglomerados con variables categóricas y categorizadas (como la edad), mediante el programa SPAD (v.4.5). Para ello, en primer lugar se realizó un análisis de correspondencias múltiples tomando como variables activas todas las relacionadas tanto con el accidente como con la víctima, descritas anteriormente. De este análisis se retuvieron los diez primeros ejes, que son combinaciones de dos o más variables nominales, que explicaban el 38,55\% de la varianza total. La clasificación se realizó según las coordenadas de los individuos en estos ejes y las clases se identificaron según los índices de nivel o medidas de las distancias entre las clases; finalmente, se caracterizaron las clases mediante los valores test que arroja el análisis, que permiten comparar la proporción de casos de la clase que presentan una característica (modalidad) con la proporción de los casos de la muestra total que presentan ese atributo (Béçue, 1991).

\section{Resultados}


Resultados generales de la encuesta a estudiantes

La tabla 1 presenta los resultados obtenidos en la encuesta para los tres indicadores de victimización por accidentalidad vial para toda la muestra y por departamento. Como se puede apreciar, de 2292 sujetos el $15,7 \%$ ha sido víctima directa de un accidente de tránsito, algo más de una cuarta parte $(27,4 \%)$ conoce a familiares o amigos que han sido heridos, y cerca del $21 \%$ conoce un familiar muerto en las vías. El análisis por departamentos se muestra en la tabla 1 , en la cual aparecen en negrita las cifras de los cinco departamentos con mayor tasa de víctimas en cada una de las tres modalidades. Estos resultados muestran que en algunos departamntos las tasas son de las más altas tanto para los heridos encuestados, como los que conocen heridos en familiares o amigos, como para los que conocen fallecidos. Así, César y Sucre presentan una alta tasas de víctimas de accidentalidad vial. Otros departamentos, como el caso de Vichada y de Caquetá presentan tasas muy altas de encuestados heridos por accidentes. En contraste, también se encuentra que hay departamentos con tasas bajas de accidentalidad en los tres indicadores. Así, la observación de la tabla 1 muestra que las muestras del Chocó, del Quindio y de Risaralda presenta las tasas más bajas de accidentalidad. Además, la muestra del departamento del Valle del Cauca presenta tasas bajas de accidentalidad en el porcentaje de encuestados heridos y en el de familiares/amigos fallecidos.

Tabla 1. Distribución de victimización por accidentalidad vial en estudiantes colombianos universitarios y del SENA (muestra total: 2292 sujetos). Eventos ocurridos dentro de los seis meses anteriores a la encuesta

\begin{tabular}{|c|c|c|c|c|c|c|}
\hline \multirow[b]{2}{*}{ Departamento } & \multicolumn{2}{|c|}{$\begin{array}{l}\text { Lesiones propias por } \\
\text { accidente vial }\end{array}$} & \multicolumn{2}{|c|}{$\begin{array}{l}\text { Lesiones por acciden- } \\
\text { te vial familiar o ami- }\end{array}$} & \multicolumn{2}{|c|}{$\begin{array}{l}\text { Muerte por accidente } \\
\text { vial familiar o amigo/a }\end{array}$} \\
\hline & $\mathrm{n}$ & $\%$ & $\mathrm{n}$ & $\%$ & $\mathrm{n}$ & $\%$ \\
\hline Amazonas & 19 & 5,4 & 21 & 3,4 & 9 & 1,9 \\
\hline Antioquia & 8 & 2,3 & 18 & 2,9 & 17 & 3,7 \\
\hline Atlántico & 20 & 5,7 & 46 & 7,5 & 38 & 8,2 \\
\hline Bolívar & 22 & 6,3 & 43 & 7 & 36 & 7,8 \\
\hline Caquetá & 41 & 11,6 & 41 & 6,6 & 25 & 5,4 \\
\hline Cesar & 34 & 9,7 & 61 & 9,9 & 43 & 9,3 \\
\hline Chocó & 2 & 0,6 & 7 & 1,1 & 8 & 1,7 \\
\hline Córdoba & 27 & 7,7 & 34 & 5,5 & 30 & 6,5 \\
\hline Cundinamarca & 17 & 4,8 & 41 & 6,6 & 29 & 6,3 \\
\hline Guainía & 19 & 5,4 & 18 & 2,9 & 13 & 2,8 \\
\hline La Guajira & 9 & 2,6 & 52 & 8,4 & 35 & 7,6 \\
\hline Magdalena & 23 & 6,5 & 41 & 6,6 & 27 & 5,8 \\
\hline Putumayo & 19 & 5,4 & 23 & 3,7 & 18 & 3,9 \\
\hline Quindío & 5 & 1,4 & 7 & 1,1 & 7 & 1,5 \\
\hline Risaralda & 2 & 0,6 & 6 & 1 & 7 & 1,5 \\
\hline Sucre & 26 & 7,4 & 85 & 13,8 & 64 & 13,8 \\
\hline Tolima & 11 & 3,1 & 13 & 2,1 & 11 & 2,4 \\
\hline Valle del Cauca & 3 & 0,9 & 14 & 2,3 & 7 & 1,5 \\
\hline Vaupés & 12 & 3,4 & 18 & 2,9 & 18 & 3,9 \\
\hline Vichada & 33 & 9,4 & 28 & 4,5 & 21 & 4,5 \\
\hline Total & 352 & 15.7 & 617 & 27.4 & 463 & 20.6 \\
\hline
\end{tabular}


Un análisis de correlación de Spearman entre los porcentajes de víctimas de los tres indicadores de la tabla 1 indica que existe una fuerte asociación entre ellos; es decir, allí donde hay un porcentaje alto de heridos por accidentalidad vial, también hay porcentajes altos de muertos, y ello tanto para los encuestados como para sus familiares y amigos. La correlación entre el número de encuestados heridos y encuestados con familiares o amigos heridos fue de .729; entre el número de encuestados heridos y familiares muertos fue de .718; y entre familiares heridos y familiares muertos fue .955. Todas las correlaciones son significativas con $\mathrm{p}<.001$.

Perfiles sociodemográficos de los heridos en accidentes

352 de los participantes reportaron haber resultado lesionados por accidente de tránsito, las edades oscilan entre los 16 y 52 años, con media en los 22.2 y desviación típica de 5 años, la proporción de mujeres (casi 55\%) es algo más del 9\% que la de hombres y predominan los estratos bajos. Además, en correspondencia con la edad, la gran mayoría (80\%) se declaró soltero, siendo la proporción de casados de tan sólo el 5\% (ver tabla 2).
En la base de datos del INML sobre heridos en el año 2009 se identificaron 1834 registros en el mismo rango de edad (16 a 52) de la muestra de participantes en el estudio. En contraste con lo encontrado en la investigación con estudiantes, la mayoría de las víctimas son hombres, doblando casi a la proporción de mujeres heridas (hombres, 62.9\%; mujeres, $37.1 \%$, para una muestra total de 1843 registros), y la media de edad (32.02 años, con desviación típica de 9.81) es mayor que la encontrada en la muestra de estudiantes. En cuanto al estado civil, la moda es ser soltero, pero esta proporción de solteros/as es casi la mitad (40.2\%) de lo encontrado en la encuesta. Le sigue, en los datos de INML, los estados de unión libre (31.4\%), estar casado/a (18.1\%), divorciado o separado (4.6\%) y viudo (0.9\%). Se encuentran además dos casos de sujetos de vida religiosa. De forma complementaria también se puede indicar que la mayoría de los sujetos de los registros del INML corresponde a sujetos con la educación secundaria completa $(44.0 \%)$ o incompleta (15.8\%), con educación primaria completa o incompleta (14.7\%) con una presencia de estudiantes universitarios del $12.3 \%$ y de estudiantes de carreras afines a las del SENA del 9.9\%.

Tabla 2. Descripción sociodemográfica de sujetos que informaron haber sufrido lesiones asociadas a accidente de tránsito $(\mathrm{n}=352)$

\begin{tabular}{llc}
\hline Variable & Categoría & Porcentaje \\
\hline \multirow{2}{*}{ Sexo } & Masculino & 54.8 \\
& Femenino & 45.2 \\
\hline \multirow{4}{*}{ Estado civil } & Soltero/a & 80.7 \\
& Unión libre & 12.3 \\
& Casado/a & 5.0 \\
& Divorciado/a & 1.2 \\
& Viudo/a & 0.9 \\
\hline \multirow{2}{*}{ Estrato socioeco- } & Uno o Dos & 74.0 \\
nómico & Tres & 18.8 \\
& Cuatro & 3.8 \\
& Cinco o seis & 3.5 \\
\hline
\end{tabular}


Cuando se seleccionan en los registros del INML los casos no sólo por la edad sino por el nivel de estudios (universitario o técnico) para obtener una submuestra más equiparable a la de la investigación con estudiantes, se obtuvo un subgrupo de 409 casos, en los cuales la proporción de hombres heridos en accidente vial es menor (48.9\%) que el de las mujeres $(51.1 \%)$; resultado semejante al de los datos de estudiantes. Ello podría deberse a que en niveles académicos superiores, la mujer tiene mayor acceso a manejar vehículos o a actividades que implican transportarse, por lo cual se expone más que en otros niveles educativos a ciertos accidentes de tránsito.

Variables asociadas con los accidentes de tránsito con heridos

Un primer resultado relacionado con las características de los accidentes indica que la gran mayoría de ellos ocurren en zona urbana, $98.2 \%$ de víctimas pertenecen al medio urbano, lo cual resulta esperado en Bogotá como gran urbe. De manera coherente, la mayoría de los sucesos ocurren en las calles (71.9\%) o en escenarios públicos (20.8\%); un 3.2\% ocurre en un vehículo de servicio público y sólo un $1.4 \%$ de casos en un vehículo particular.

Un aspecto sumamente importante para comprender los factores de riesgo de la accidentalidad vial es la descripción de las circunstancias del hecho. Sin embargo, en la base de datos analizada la respuesta más frecuente sobre las circunstancias fue la categoría "Otras" (47\%) y categorías como "Violaciones de otras normas de tránsito" $(7,3 \%)$, las cuales son poco informativas. En suma, se carece de información útil y práctica sobre las circunstancias del accidente en más del $70 \%$ de los casos de accidentes con heridos. Por otro lado, dentro de este aspecto y con categorías más informativas, se encuentra que el $7.5 \%$ de los casos se atribuye a exceso de velocidad y el $2 \%$ a violación de normas de tránsito por parte de peatones. En cuanto a diferencias entre grupos, el exceso de velocidad está menos presente $(5.1 \%$ de los casos) en los conductores de 16 a 52 años con estudios avanzados (universitarios o técnicos) que en los demás (8.3\%).

El tipo de vehiculo más frecuentemente involucrado en el este tipo de accidentalidad son los automóviles, incluyendo taxis (23.1\%), las motos o motocarros (23.3\%), las busetas (16.9\%) y los buses (14.8\%). Comparando los dos grupos de víctimas, entre quienes tienen estudios superiores $\mathrm{O}$ técnicos y edades de 16 a 52 años, es mayor la proporción de accidentes con participación de automóviles-taxis (32.6\% vs. $18.3 \%$ ) y de motos o motocarros (31.3\% vs. 19.3\%), mientras que los sujetos con niveles educativos bajos presentan mayor accidentalidad en bicicletas (16.8\% vs. $4.6 \%$ del primer grupo) y buses (17.3\% vs. $9.9 \%$ ). De nuevo, como en el caso anterior, esta diferencia entre grupos podría ligarse con el mayor acceso a vehículos de motor del grupo con niveles más altos de estudio. La comparación de proporciones muestra resultados estadísticamente significativos (Chi2=68.00, p <.001). La comparación por género también arroja resultados significativos con Chi2 de 315.18 y p <.001. Los hombres son más involucrados en accidentes con bicicleta (15.2\% vs. 3.8\%) y con moto o motocarro (44.7\% vs. $14.2 \%)$, mientras que las mujeres presentan mayor proporcion de accidentes con automóviles $(24.3 \%$ vs. $18.0 \%)$ y buses, busetas y microbuses (50.9\% vs. $16.8 \%$ ).

El tipo de accidente más frecuente es el atropello (41.3\%) sobre todo en los sujetos de menores niveles educativos (47.1\%) en comparación con los universitarios/técnicos de 16 a 52 años (25.3\%). En segundo lugar se encuentran los choques entre vehículos 
(35.4\%), con proporciones casi inversas entre los dos grupos comparados, $32.2 \%$ para niveles educativos bajos y $44 \%$ para niveles educativos altos (Chi2 $=77.08$, p $<.001$ ). Otro tipo frecuente de accidentalidad es la caída del ocupante, que se da en el $11.9 \%$ de los casos analizados. En cuanto al género, en las mujeres se encuentra con mayor proporción el atropello (40.2\%) y la caída del ocupante (18.1\%) mientras que en los hombres el atropello se reporta en un $34.7 \%$ y la caída del ocupante en un $7.8 \%$; en éstos últimos es más frecuente el choque con otro vehículo en un $46.4 \%$ de los casos y sólo el $28.7 \%$ en las mujeres $($ Chi2 $=109.30, \mathrm{p}<.001)$.

Los resultados del análisis de variables relacionadas con las víctimas muestran que la mayoría de ellas eran peatones (41.2\%) o pasajeros del vehículo (35.7\%) y en un $23.1 \%$ de los casos, la víctima fue el conductor del mismo. Sin embargo, dentro de la muestra de sujetos con estudios técnicos/universitarios y de edades entre 16 a 52 años, es mayor la proporción de víctimas conductores (29.9\% vs. 20.6\%) y de pasajeros (44.9\% vs. $32.4 \%$ ) mientras que para el resto de casos analizados, es mayor la proporción de víctimas peatones $(47.0 \%$ vs. $25.2 \%)$. Este resultado podría explicarse por el acceso diferencial al manejo de vehículos de motor asociado al mayor ingreso económico que, a su vez, puede asociarse a un mayor nivel nivel educativo. En cuanto al género, el resultado es contundente con un Chi2 de 426.75, y p $<.001$, mostrando que la proporción de víctimas conductores hombres (43.0\%) es muy superior al de las mujeres (6.7\%), siendo entonces las mujeres más víctimas que los hombres como pasajeras ( $53.1 \%$ vs. $22.4 \%$ y en menor medida como peatones (40.2\% vs. $34.6 \%)$.

La información sobre la actividad de la víctima en el momento del accidente es muy deficiente en las bases de datos oficiales, ya que un altísimo porcentaje (61.7\%) de casos están categorizados en "otras actividades". Otras categorías que arrojan algo de información se refieren a actividades de descanso o mientras se duerme o come (5.3\%) y al transporte desde o hacia el lugar de trabajo (3.5\%). Por sexos, la diferencia más marcada es que entre las mujeres es mayor la proporción de quienes se accidentaron en situaciones de dormir, descansar o comer (5.7\%) que entre los hombres $(4.3 \%$, Chi2=31.65, p<.01), quizá por que su rol, en los accidentes, es más de pasajeras.

Finalmente, en lo que se refiere al tipo de daño que el accidente ocasionó en las víctimas, el más frecuente es el trauma de miembros en un $43.2 \%$ de los casos y el politrauma con $31.4 \%$; el trauma de cuello se presenta en un $3.3 \%$ de los casos. Se destacan en esta variable, dos aspectos: el $4.7 \%$ de los casos no presenta lesiones y existen diferencias entre algunos grupos de víctimas: los sujetos de nivel educativo alto presentan mayor proporción de traumas de cuello $(7.8 \%$ vs. $1.7 \%)$ y de trauma de miembros $(46.0 \%$ vs. $42.2 \%)$ y menos politrauma (26.2\% vs. $33.3 \%)$, trauma craneano (2.4\% vs. $3.8 \%)$ o facial $(6.8 \%$ vs. 9.7\%) que los otros sujetos (Chi2 $=54.60, \mathrm{p}$ $<.001$ ). En cuanto al género, en las mujeres es más frecuente el trauma de cuello $(4.5 \%$ vs. $1.8 \%$ ) y en los hombres el trauma de miembros $(47.2 \%$ vs. $41.0 \%$ en mujeres, Chi2=37.02, $\mathrm{p}<.001)$.

\section{Tipologías de accidentes}

Como resultado del Análisis de Correspondencias Múltiples se identificaron los diez primeros ejes que explicaban el 38,55\% de la varianza y el análisis de clasificación sobre los mismos permitió identificar cuatro clases. 
Una variable que pesa de manera importante en la identificación de las cuatro clases es la edad de la víctima. Como puede verse en la tabla 3, la primera clase incluye de manera significativa a las personas mayores de 60 años, la segunda reune a los niños menores de 10 años y la cuarta a las víctimas de edad media, entre 20 y 30 años. Así, las variables relacionadas con el accidente parecen ser diferentes para cada uno de estos grupos de edad.

Tabla 3. Tipologías de accidentes de tránsito en Bogotá. Clasificación jerárquica.

\begin{tabular}{|c|c|c|c|}
\hline Variable - categoría & $\begin{array}{l}\text { \% casos en la clase } \\
\text { con la característica }\end{array}$ & $\begin{array}{c}\text { \% de casos con la } \\
\text { característica en el } \\
\text { conjunto de la muestra }\end{array}$ & Valor Test. \\
\hline \multicolumn{4}{|l|}{ Tipología $1(\mathrm{n}=781)$} \\
\hline Atropello & 99,10 & 36,85 & $47,61^{* * *}$ \\
\hline Peatón como víctima & 98,98 & 36,81 & $47,50 * * *$ \\
\hline Vehículo particular & 46,99 & 29,64 & $12,68 * * *$ \\
\hline Edad víctima: 60 años en adelante & 12,42 & 7,50 & $6,07 * * *$ \\
\hline \multicolumn{4}{|l|}{ Tipología $2(\mathrm{n}=193)$} \\
\hline Edad víctima: 0 a 10 años & 66,32 & 5,34 & $27,24^{* * *}$ \\
\hline Primaria incompleta & 30,57 & 7,50 & $10,08 * * *$ \\
\hline Víctima peatón & 56,99 & 36,81 & $5,87^{* * *}$ \\
\hline Atropello & 56,99 & 36,93 & $5,86 * * *$ \\
\hline Lesiones: trauma facial & 15,03 & 8,92 & $2,79 * *$ \\
\hline \multicolumn{4}{|l|}{ Tipología $3(n=736)$} \\
\hline Víctima pasajero & 87,77 & 34,72 & $37,12 * * *$ \\
\hline Vehículo transporte público & 58,02 & 26,51 & $22,67 * * *$ \\
\hline Buseta & 28,53 & 9,09 & $21,80 * * *$ \\
\hline Automóvil & 33,97 & 12,88 & $19,68^{* * *}$ \\
\hline Caída del ocupante & 28,13 & 11,92 & $15,52 * * *$ \\
\hline Víctima mujer & 61,55 & 40,18 & $14,10^{* * *}$ \\
\hline Lesiones: trauma cuello & 8,30 & 2,88 & $10,05^{* * *}$ \\
\hline \multicolumn{4}{|l|}{ Tipología $4(\mathrm{n}=689)$} \\
\hline Víctima conductor & 81,13 & 28,43 & $36,17^{* * *}$ \\
\hline Moto o motocarro & 67,78 & 20,88 & $35,41 * * *$ \\
\hline Choque con otro vehículo & 77,65 & 39,42 & $24,58 * * *$ \\
\hline Conductor hombre & 87,08 & 59,82 & $18,19 * * *$ \\
\hline Bicicleta & 22,06 & 6,84 & $17,97 * * *$ \\
\hline Edad: 21-30 años & 45,28 & 29,51 & $10,49 * * *$ \\
\hline Unión libre & 40,49 & 25,89 & $10,07 * * *$ \\
\hline Secundaria completa & 50,94 & 36,18 & $9,40 * * *$ \\
\hline Lesiones: trauma miembros & 52,69 & 44,73 & $4,92 * * *$ \\
\hline
\end{tabular}

En la primera clase de accidentes (tipología 1) queda incluido el $32,8 \%$ y es el que ocurre principalmente a personas mayores de 60 años; consisten en atropellos a peatones por vehículos particulares aunque no hay información sobre el tipo de vehículo involucrado en el accidente: automóvil, moto, bus o camión.
La segunda tipología incluye el 8,02\% de los casos y tiene como blanco a los niños de corta edad -por lo cual se asocia a un bajo nivel educativo-, en accidentes de atropello y en los que suele haber unos daños a nivel de trauma facial. 
En cambio, las mujeres en papel de pasajeros son las victimas de la tercera tipología con el 30,6\% de los casos. En más de la mitad de los casos se transportan en vehículos de servicio público como busetas o buses y la dinámica del accidente en casi $30 \%$ de los casos es la caída desde el vehículo por parte del pasajero. En esta agrupación suele presentarse con mayor frecuencia que en otros accidentes las lesiones en el cuello.

Por último, la cuarta clase reune el 28,6\% de los casos que son mayoritariamente hombres jóvenes ( $2^{\circ}$ a 30 años) con estudios secundarios y que se encuentran conduciendo motos, motocarros o bicicletas. La dinámica del accidente suele consistir en choque con otro vehículo, con lesiones en los miembros.

\section{Discusión}

En primer lugar, los resultados muestran algunos contrastes entre los datos oficiales del Instituto de Medicina Legal y Ciencias Forenses y los obtenidos por la encuesta de victimización a estudiantes universitarios y de institutos técnicos y tecnológicos. Así hay que recordar que con frecuencia las encuestas de victimización reflejan una incidencia de la criminalidad mayor a la registrada oficialmente. En el caso de los accidentes, se encuentra que la encuesta a los estudiantes muestra un $15.7 \%$ de sujetos que auto-informan de heridas o lesiones en accidentes viales. Esta cifra es mayor que las estadísticas oficiales, pero explicable, por ejemplo, en el sentido de que sólo los accidentes graves, que generan algún tipo de lesión llegan al INML, precisamente para certificar tal daño, con fines penales o de indemnización. Por otro lado, la proporción parecida de mujeres y hombres accidentados con heridas -para el mismo grupo etáreo y de niveles educati- vos- en los datos de INML y la encuesta a estudiantes contribuye a validar los datos obtenidos en ésta.

Segundo, respecto a las características de los accidentes con heridos a partir de la base de datos del INML el primer dato que se observa es la falta de información en muchos casos en aspectos críticos, que podrían ayudar a conocer mejor la fenomenología de los accidentes de tránsito. Por ejemplo, de un total de 2399 casos, no se conoce información sobre el nivel educativo del accidentado en 116 casos, y no se conoce su estado civil en 218 , no se conoce la zona del hecho (rural o urbana) en 35 casos, y el escenario en 50. En las variables "Tipo de actividad de la víctima en el momento del accidente" y "Circunstancias del hecho", la información faltante (No sabe o no responde) o ambigua y poco informativa (Otras) afecta a más del 60\% de los casos. En suma, se carece de información útil y práctica sobre las circunstancias del accidente en más del $70 \%$ de los casos de accidentes con heridos. La ausencia de esta información en tantos casos debe llamar la atención de los responsables públicos acerca de cómo y con qué calidad se está recogiendo dicha información y se está atendiendo a las víctimas de accidente vial.

A pesar de esta limitación, la información disponible permite identificar patrones generales como las calles como escenario principal de los accidentes o el mecanismo contundente como característico de los accidentes con heridos. También permite analizar e identificar diferencias de género, por nivel educativo y edad. Así, el mayor nivel educativo se asocia con mayor acceso a vehículos a motor y con mayor exposición de las mujeres a la accidentalidad vial, mientras que un menor nivel educativo se asociaría a más accidentes de tipo atropello con peatones como víctimas, y con bicicletas y buses como vehículos involucrados. 
En los sujetos de mayor nivel educativo y edades de 16 a 52 años se presenta más el choque con otros vehículos, el conductor y pasajero como víctima y, lógicamente, la participación de vehículos de motor. En cuanto al género, las mujeres aparecen más víctimas como pasajeras o peatones, en carros, buses, busetas o microbuses en forma de atropello o caída del ocupante, mientras que las víctimas hombres son con más frecuencia conductores, involucrados en choques con otros vehículos con mayor participación de bicicletas, motos o motocarros. Para el caso de los peatones, el accidente vial no se asocia en el presente estudio con el sexo, a diferencia de lo encontrado en estudios realizados en otros contextos (Damsere, Derry, Ebel, Mock, Afukaar E Donkor, 2009).

\section{Conclusiones}

Como se indicó más arriba, recolectar información precisa sobre los factores involucrados en los accidentes viales permitiría diseñar políticas de prevención realistas y contribuir a evaluar las responsabilidades penales, civiles y de seguros a las que hubiere lugar en cada caso.

La combinación de múltiples variables de los datos oficiales de accidentalidad en Bogotá, permite identificar unas tipologías de accidentes, que indican la mayor vulnerabilidad de ciertos usuarios de la vía -niños, adultos mayores, peatones, conductores de motos o de bicicletas- a los accidentes, en las interacciones con los vehículos de mayor potencia (automóviles, buses). Es por ello que es necesario volver a señalar, siguiendo a Montoro et al. (2000), la responsabilidad que implica conducir un vehículo a motor. De otro lado, también la formación de los conductores de transporte público y su estilo de conducción se hacen visibles, al ligarse a la accidentalidad de los pasajeros, especialmente en el caso de las mujeres.

Otro hallazgo relevante es que el tipo de accidente vial se asocia con mayor probabilidad a un tipo de lesiones, y ello puede ser tenido en cuenta para la investigación que deben adelantar las autoridades judiciales para evaluar los daños (Vargas, Cuervo, E Malaver, en prensa). Por su parte, el tipo de usuario de la vía (peatón, motociclista, chófer) también se asocia con una dinámica de accidentalidad específica.

Así, el análisis de conglomerados se muestra útil no sólo para identificar tipologías de conductores (Marengo. Settani, E Viotto, 2012) o de actitudes hacia las normas de conducir y tipos de daños causados en accidentes viales (Ruiz, Beltrán, Gómez, Lamus y Leal, 2014), sino también para conocer las dinámicas de la accidentalidad vial, ya que permite combinar información de múltiples variables (Miethe E McCorkle, 2001; Morales, 2003). El hecho de que los peatones aparezcan como uno de los grupos de víctimas viales más frecuentes debería llevar al desarrollo y refuerzo de políticas de protección de este colectivo (Pucher \& Dijkstra, 2003), con políticas diferenciales para hombres y mujeres (OMS, 2002, Social Issues Research Center, 2004) y niños/as. Con relación a ello, en el Reino Unido se ha estimado que el 50\% de las muertes entre los 10 y 15 años por agentes externos tiene como causa los accidentes de tráfico (RAC, s/f). En esta línea, se podría plantear dar prelación en la movilidad vial en medios urbanos masificados a peatones y ciclistas. 


\section{Referencias}

Béçue, M. (1991). Análisis de datos textuales. París. Cisia. Łlink〕

CESVI-Colombia. (2003). Boletín de Seguridad. Mayo 2003.

Damsere-Derry, J., Ebel, B.E., Mock, C.N., Afukaar, F., E Dokor, P. (2009). Pedestrians injury patterns in Ghana. Accident Analysis and Prevention, 42(4), 1080-1098. DOI:10.1016/j.aap.2009.12.016. link]

Informe de Desarrollo Humano para Bogotá. (s.f). El futuro de la movilidad en Bogotá. Bogotá: El Malpensante. Extraído el 6 de enero del 2009 de http://www.idhbogota.pnud.org.co/cuadernos/Movilidad.pdf

Kadali, B.R. E Vedagiri, P. (2013). Effect of vehicular lanes on pedestrian gap acceptance behavior. Procedia - Social and Behavioral Sciences. 104, 678-687. DOI: 10.1016/j.sbspro.2013.11.162. _link」

Kaiser, G. (1978). Delincuencia de tráfico y su prevención general. Colección Estudios de Psicología Criminal. Vol. XIX. Madrid: Espasa-Calpe.

Koh, P.P. \& Wong, Y.D. (2014). Gap acceptance of violators at signalized pedestrian crossings. Accident Analysis \& Prevention. 62, 178-185. DOI: 10.1016/j.aap.2013.09.020.」link」

Marengo, D., Settani, M., \& Vidotto, G. (2012). Drivers' subtypes in a sample of Italian adolescents: relationship between personality measures and driving behavior. Transportation Research Part F. 15, 480-490. DOI: 10.1016/j.trf.2012.04.001 Џlink

Martin, J.L., Lafont, S., Chiron, M., Gadeqbeku, B., E Laumon, B. (2004). Differences between males and females in traffic accident risk in France. Rev Epidemiol Sante Publique, 52(4), 357-67. «link

Mayou, R., E Bryant, B. (2010). Consequences of road traffic accidentes for different types of road user. Injury, 34, 197-202. DOI: 10.1016/S0020-1383(02)00285-1 Џlink

Miethe, T.D., \& McCorkle, R.C. (2001). Crime profiles: The Anatomy of Dangerous Persons, Places and Situations. Los Angeles: Roxbury Publications Company. «link」

Montoro, L., Alonso, F., Esteban C., E Toledo, F. (2001) Manual se seguridad vial: El factor Humano. Barcelona: Ariel. [link]

Morales, L.A. (2003). La técnica del perfil en la investigación criminal. En V.Garrido (Ed.). Psicópatas y otros delincuentes violentos (305-390). Valencia: Tirant. 〔link」

Organización Panamericana de Salud. (s.f.). Informe Mundial sobre prevención de los traumatismos y causados por el tránsito: elementos para la toma de decisiones. Recuperado el 14 de noviembre del 2010. _link」

Organización Mundial de la Salud. (2002). Gender and road traffic injuries. Ginebra: Department of Gender and Women's Health. 〔link」

Organización Mundial de la Salud. (2003). Informe sobre la salud en el mundo 2003. Ginebra. 〔link」

Organización Mundial de la Salud. (2009). Informe sobre la situación mundial de la seguridad vial: es hora de pasar a la acción. Ginebra. Цlink」

Páez, D. E Vergara, A.I. (2000). Theoretical and methodological aspects of cross-cultural research. Psicothema, 12(2), 1-5. «link」 
Pucher, J. E Dijkstra, L. (2003). Promoting safe walking and cycling to improve public health: Lessons from The Netherlands and Germany. Public Health Problems, 93(9), 1509-1516. ¿link」

RAC. (s/f). Mortality and road traffic accidents in the UK. Recuperado de http://www.racfoundation.org/assets/rac foundation/content/downloadables/road\%20accident $\% 20$ casualty\%20comparisons\%20-\%20box\%20-\%20110511.pdf, el 16 de diciembre de 2015. [link」

Rodríguez, M.A, (2009). Los efectos del control externo e interno en la prevención de la conducción antisocial. V Congreso Nacional de Psicología Jurídica y Forense. Granada, 19-21 de noviembre del 2009.

Ruiz, J.I. E López, C. (2010). Escala de dificultades percibidas para la conducción, hostilidad y extraversión: un análisis correlacional en conductores de Bogotá. Diversitas: Perspectivas en Psicología, 6(2), 441-454. link」

Ruiz, J.I., López, E.J., Norza, E., \& Rodríguez, L.S. (2012). Social y Jurídica, vol. 2.: Monográfico Percepción de Seguridad en Jóvenes Colombianos.

Ruiz, J.I. (2013). Escala de Dificultades en la Conducción: Fiabilidad y Validez en una muestra de conductores de Colombia. Congreso Internacional de Seguridad Vial. Santander, España, 16-18 mayo 2013.

Ruiz, J.I., Beltrán, I.T., Gómez, I.A., Lamus, D.A., \& Leal, L.J. (2014). Representaciones sociales de normas de tránsito, agresividad, facilidad percibida en la conducción, accidentes y multas en conductores de Bogotá D. C. Revista Criminalidad. Łlink]

Ruiz, J.I. (2015). Resiliencia comunitaria: propuesta de una escala y su relación con indicadores de violencia criminal. Pensamiento Psicológico, 13(1), 119-135. «link」

Schwebel, D.C., Stavrinos, D., E Kongable, E.M. (2009). Attentional control, high intensity pleasure and risky pedestrian behavior in collage students. Accident Analysis and Prevention, 41, 658.661. DOI: 10.1016/j.aap.2009.03.003 [link〕

Sharkin, B. (2004). Road Rage: Risk Factors, Assessment, and Intervention Strategies. Journal of Counseling \& Development, 82, 191-198. DOI: 10.1002/j.1556-6678.2004.tb00301.x. 〔link」

Sivak, M. E Schoettle, B. (2011). Toward understanding on-road interactions of male and female drivers. Traffic Injury Prevention, 12, 235-238. [link]

Social Issues Research Center. (2004). Sex differences in driving and insurance risk. Oxford: SIRC.

Vargas, H.O., Cuervo, A., E Malaver, I.M. (en prensa). Detección del fraude en seguros de vehículos. En J.I.Ruiz, H.O.Vargas, \& L.M. Chacón (2014). Psicologia Juridica y Evaluacion del Testimonio en la Investigacion del Fraude. Editorial UN.

Yagil, D. (2000). Beliefs, motives and situational factors related to pedestrians' self-reported behavior at signal-controlled crossings. Transportations Research. F3, 1-13. DOI: 10.1016/S13698478(00)00004-8. [link]

Recibido: Junio 4-2015 Revisado: Diciembre 7-2015 Aceptado: Marzo 9-2016 